\title{
ARTICLE \\ Clinical, behavioral, and neural measures of reward processing correlate with escitalopram response in depression: a Canadian Biomarker Integration Network in Depression (CAN-BIND-1) Report
}

\author{
Katharine Dunlop (D), Sakina J. Rizvi ${ }^{2,3,4}$, Sidney H. Kennedy ${ }^{2,3,4,5}$, Stefanie Hassel ${ }^{6}$, Stephen C. Strother (D) $^{7}$, Jacqueline K. Harris ${ }^{8}$, \\ Mojdeh Zamyadi ${ }^{7}$, Stephen R. Arnott ${ }^{7}$, Andrew D. Davis ${ }^{9}{ }^{9}$, Farrokh Mansouri ${ }^{10}{ }^{10}$, Laura Schulze ${ }^{2}$, Amanda K. Ceniti ${ }^{2,3}$, \\ Raymond W. Lam (iD ${ }^{11}$, Roumen Milev ${ }^{12,13}$, Susan Rotzinger ${ }^{3,4,5}$, Jane A. Foster ${ }^{5,9}$, Benicio N. Frey (iD ${ }^{9,14}$, Sagar V. Parikh ${ }^{15}$, \\ Claudio N. Soares ${ }^{12}$, Rudolf Uher ${ }^{16}$, Gustavo Turecki $\mathbb{D}^{17}$, Glenda M. MacQueen ${ }^{6}$ and Jonathan Downar $\mathbb{I D}^{2,4,5}$
}

\begin{abstract}
Anhedonia is thought to reflect deficits in reward processing that are associated with abnormal activity in mesocorticolimbic brain regions. It is expressed clinically as a deficit in the interest or pleasure in daily activities. More severe anhedonia in major depressive disorder (MDD) is a negative predictor of antidepressant response. It is unknown, however, whether the pathophysiology of anhedonia represents a viable avenue for identifying biological markers of antidepressant treatment response. Therefore, this study aimed to examine the relationships between reward processing and response to antidepressant treatment using clinical, behavioral, and functional neuroimaging measures. Eighty-seven participants in the first Canadian Biomarker Integration Network in Depression (CAN-BIND-1) protocol received 8 weeks of open-label escitalopram. Clinical correlates of reward processing were assessed at baseline using validated scales to measure anhedonia, and a monetary incentive delay (MID) task during functional neuroimaging was completed at baseline and after 2 weeks of treatment. Response to escitalopram was associated with significantly lower self-reported deficits in reward processing at baseline. Activity during the reward anticipation, but not the reward consumption, phase of the MID task was correlated with clinical response to escitalopram at week 8 . Early (baseline to week 2) increases in frontostriatal connectivity during reward anticipation significantly correlated with reduction in depressive symptoms after 8 weeks of treatment. Escitalopram response is associated with clinical and neuroimaging correlates of reward processing. These results represent an important contribution towards identifying and integrating biological, behavioral, and clinical correlates of treatment response. ClinicalTrials.gov: NCT01655706.
\end{abstract}

Neuropsychopharmacology (2020) 45:1390-1397; https://doi.org/10.1038/s41386-020-0688-x

\section{INTRODUCTION}

Anhedonia, a persistent reduction in interest or pleasure in daily activities, is a core clinical feature of MDD that can be studied from clinical, behavioral, and neurobiological perspectives. Anhedonia subsumes three core maladaptive behaviors: (1) dysfunction in motivated behavior for a reward (wanting/anticipatory); (2) reduction in experience of pleasure (liking/consummatory); and (3) disrupted reward learning [1]. Several studies have independently reported that greater pre-treatment behavioral and clinical deficits in reward learning and anhedonia are related to poor acute antidepressant response [1], long-term antidepressant response [2], and non-invasive brain stimulation response [3].
The possibility that anhedonia and its biological substrates might predict treatment response in MDD is important because although numerous treatment strategies are available, only one-third of patients remit during their first intervention, and the probability of achieving remission declines with each successive monotherapy $[4,5]$. Consequently, a major goal of current psychiatric research is to identify markers that increase the likelihood of predicting who will respond to a given antidepressant treatment.

Many of the neurobiological underpinnings of anhedonia have been observed during behavioral paradigms such as the monetary incentive delay (MID) task. Studies of the MID task in MDD support an association between deficits in reward anticipation and lower

\footnotetext{
${ }^{1}$ Department of Psychiatry, Weill Cornell Medicine, New York, NY, USA; ${ }^{2}$ Institute of Medical Science, University of Toronto, Toronto, ON, Canada; ${ }^{3}$ ASR Suicide and Depression

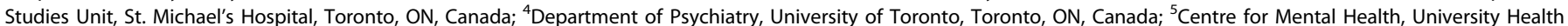

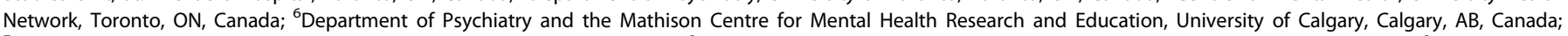
${ }^{7}$ Rotman Research Institute, Baycrest Health Sciences, Toronto, ON, Canada; ${ }^{8}$ Department of Computer Science, University of Alberta, Edmonton, AB, Canada; ${ }^{9}$ Department of

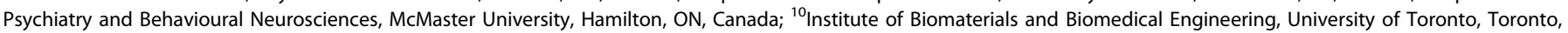

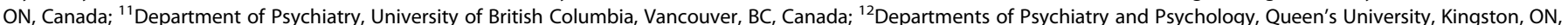

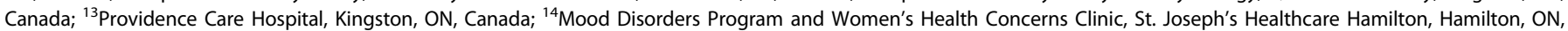

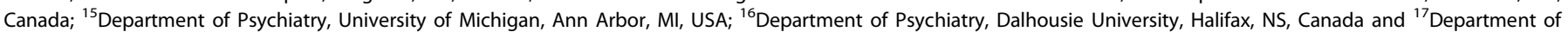
Psychiatry, McGill University, Montreal, QC, Canada

Correspondence: Katharine Dunlop (kad2032@med.cornell.edu)
}

Published online: 29 April 2020 
activity in frontostriatal networks including the anterior cingulate cortex (ACC) and middle/medial frontal gyri [6-9]. Deficits during reward consumption have been associated with decreased activity in basal ganglia [10-13], which, independent of diagnosis, may be related to underlying dopaminergic $[7,13]$ and serotonergic dysfunction [14]. Individuals with MDD also display substantial heterogeneity in relation to anhedonia, possibly reflecting biological subtypes of the disorder [15], and possibly accounting for why not all studies report significant differences between control and MDD participants during reward processing [16].

There is also further evidence that this heterogeneity in clinical and biological markers of reward processing may predict response to antidepressant treatments. For example, one study recently identified four MDD 'biotypes' related to anhedonia and anxiety, with biotype membership and its corresponding abnormal functional connectivity (FC) predicting treatment responsiveness to non-invasive brain stimulation with $>85 \%$ accuracy [15]. High or sustained frontostriatal connectivity or activity stemming from the ventral striatum (VS) during anticipation or receipt of reward is associated with response to psychotherapy [16-18] and with adjunctive pharmacotherapy [19].

Pharmacotherapy-induced changes in frontostriatal responses during reward processing in MDD appear rapidly. Increased frontostriatal connectivity and striatal activity during reward processing was observed following a single psychotropic dose relative to placebo [20], and caudate resting-state dynamics change within $5 \mathrm{~h}$ of escitalopram administration [21]. Such increases in reward sensitivity related to antidepressant response and caudate function persist at 12 weeks of treatment [22]. Crucially, our group recently reported that self-reported improvements in reward sensitivity within the first 2 weeks of escitalopram treatment predicted later response [23]. It is not fully understood whether escitalopram response is related to early changes in frontostriatal activity during reward preprocessing.

The aim of this study is to determine whether the clinical, behavioral, and neural circuitry correlates of reward processing predict antidepressant response to 8 weeks of escitalopram, a first-line pharmacotherapy. In response to previous work [1-3], we hypothesized that clinically greater baseline severity of selfreported anhedonia would be associated with poor escitalopram response. We also predicted that task-related response time would be inversely correlated with symptom improvement. Consistent with previously published work in psychotherapy [17], we hypothesized that lower baseline frontostriatal activity and connectivity during the reward anticipation and consumption phases of a reward processing task would correlate with response to escitalopram. As in previous studies and in a recent report from our group assessing the utility of early change in symptom severity as a mediator of antidepressant response $[24,25]$, we predicted that early (baseline to week 2 of treatment) increases in frontostriatal activity and connectivity during the same task would correlate with later escitalopram response after 8 weeks of treatment. Lastly, given that baseline correlates of reward processing have been shown to predict early treatmentassociated changes [23], we performed an exploratory multiple linear regression model to examine the relationships between baseline predictors and early frontostriatal change with later escitalopram response.

\section{METHODS}

Participants

Participants were recruited at six academic sites across Canada as part of the first Canadian Biomarker Integration Network in Depression protocol (CAN-BIND-1). The overarching goal of CAN-BIND-1 was to identify and integrate biomarkers of response to antidepressant monotherapy and adjunctive therapy [25] using neuroimaging, electrophysiology, neurocognitive testing, and genomic, transcriptomic, and proteomic profiling. Briefly, participants in the MDD group had a current Major Depressive Episode and scored 24 or higher on the Montgomery-Åsberg Depression Rating Scale (MADRS) [26]. In addition, healthy control (HC) participants with no psychiatric history were recruited and completed the same assessment protocol without receiving antidepressant medication. Details of the full trial protocol are described elsewhere [25] and in Supplementary Materials. All participants gave informed and voluntary consent, and the study was approved at each site by its respective Research Ethics Board. Here we include participants who completed clinical measures of anhedonia and functional neuroimaging designed to evaluate brain activity associated with reward processing.

\section{Treatment}

The protocol and main clinical outcomes have been described previously [25]. Briefly, 211 eligible MDD participants completed a baseline visit and initiated open-label treatment with escitalopram (10-20 mg daily) for 8 weeks, at a flexible dose based on clinical improvement and tolerability. Of these participants, $180 \mathrm{com}$ pleted the week 8 visit. A HC group $(n=112)$ completed the same protocol but did not receive escitalopram and served as a comparator group (ClinicalTrials.gov: NCT01655706).

\section{Assessments}

The MADRS was used to assess baseline symptom severity and change during treatment. Change in MADRS score was treated as a continuous variable, defined as the percent change in MADRS from baseline to week 8. Two self-report questionnaires were used to characterize anhedonia: the Snaith-Hamilton Pleasure Scale (SHAPS) [27], which consists of 15 items, with higher scores reflecting greater consummatory anhedonia; and the Dimensional Anhedonia Rating Scale (DARS) [28], a 17-item questionnaire that assesses four domains of hedonic function, with lower scores indicating more severe deficits in these domains. Both the DARS and SHAPS were completed at baseline and at week 8 by all participants.

\section{MRI acquisition}

High-resolution anatomical and task-based fMRI data were collected at six sites. During neuroimaging, MDD and HC participants completed either the MID task discussed in this manuscript, or an emotional conflict task reported elsewhere [29]. Of the $117 \mathrm{MDD}$ and $52 \mathrm{HC}$ participants who completed the MID task at baseline, scans of $87 \mathrm{MDD}$ and $46 \mathrm{HC}$ participants met the fMRI quality assurance standards outlined in Supplementary Materials.

The MID task is a behavioral paradigm used to assess anticipatory and consummatory responses to reward $[8,12,30-$ 32] (Supplementary Materials). Briefly, participants are presented with a visual cue indicating whether the current trial is or is not associated with a monetary reward. Only successfully executed trials with a monetary incentive cue are rewarded. This version of the MID task did not include monetary loss trials. Furthermore, the magnitude of reward during incentivized trials did not change across trials. Neuroimaging was acquired at baseline, and after 2 and 8 weeks of escitalopram treatment. Acquisition parameters and standardization procedures across sites are available in Supplementary Materials and is published elsewhere [33].

fMRI preprocessing \& statistical analysis

Site differences in clinical and behavioral data were assessed at both timepoints (baseline and week 2) and were included as covariates in a partial correlation analysis to establish the relationships between clinical or behavioral measures and percent escitalopram improvement. All partial correlations were performed using bootstrapping with 10,000 iterations using SPSS (version 21, IBM Corp., Chicago, USA). 
Preprocessing of fMRI data was performed using FSL (v5.0.10) [34] and is summarized in Supplementary Materials. Individuallevel statistics were generated using an event-related general linear model design. All stimuli and responses were modeled as explanatory variables and convolved to the double-gamma blood-oxygen-level dependent (BOLD) response function. Similar to previous studies [12, 17, 20, 35], a generalized psychophysiological interaction analysis was also performed at the single-subject level using the time-series from a VS region of interest extracted from the Harvard-Oxford subcortical atlas [36] (Supplementary Materials). The VS was selected as the only ROI due to its well-established role in reward processing during this paradigm, and its task-based FC during reward processing has been implicated in psychotherapy response [12, 17, 20]. VS-FC was modeled as an explanatory variable along with other stimulus/response regressors at the single-subject level and an interaction variable corresponding to the interaction of

Table 1. Summary of demographic and clinical measures for $\mathrm{HC}$ and MDD participants.

\begin{tabular}{|c|c|c|c|c|}
\hline & \multicolumn{2}{|c|}{ Healthy controls } & \multicolumn{2}{|l|}{ MDD } \\
\hline & Mean & SD & Mean & SD \\
\hline$n$ Female & 29 & - & 58 & - \\
\hline$n$ Right-handed & 37 & - & 79 & - \\
\hline Age & 35.31 & 12.22 & 33.61 & 11.46 \\
\hline \multicolumn{5}{|l|}{ MADRS } \\
\hline Baseline & 0.89 & 1.73 & 29.53 & 5.23 \\
\hline Week 2 & 0.79 & 1.55 & 21.99 & 7.62 \\
\hline Week 4 & - & - & 17.97 & 8.85 \\
\hline Week 6 & - & - & 15.54 & 8.52 \\
\hline Week 8 & 1.14 & 2.04 & 15.00 & 9.12 \\
\hline Percent improvement & - & - & 49.54 & 30.05 \\
\hline \multicolumn{5}{|l|}{ SHAPS } \\
\hline Baseline & 1.36 & 2.05 & 6.93 & 3.64 \\
\hline Week 8 & 0.72 & 1.16 & 4.06 & 3.77 \\
\hline \multicolumn{5}{|l|}{ DARS } \\
\hline Baseline & 59.65 & 9.10 & 32.98 & 13.45 \\
\hline Week 8 & 60.21 & 7.79 & 43.53 & 16.83 \\
\hline
\end{tabular}

whole-brain VS-FC to reward anticipation (incentivized $>$ nonincentivized cues) and reward consumption (rewarded $>$ neutral feedback).

Group-level statistics were performed using a mixed-effects general linear model to identify significant differences in the hemodynamic response during reward anticipation and consumption (incentivized $>$ non-incentivized cues; rewarded $>$ neutral feedback) and the interaction in VS-FC during the same conditions between MDD and HC groups. Group-level statistics were used to identify baseline and early change (baseline to week 2) associated with response using a linear regression analysis, based on percent MADRS improvement from baseline to week 8 . All group-level statistics accounted for recruitment site, age, sex, and baseline MADRS severity. The resultant group-level maps were clustercorrected by a height threshold of $p<0.001$, with a corrected cluster significance threshold of $p<0.05$, two-tailed. For the four contrasts assessing predictors of outcome at either baseline or during early (baseline to week 2) change, we further thresholded the statistics at a cluster significance of $p<0.05 / 8$, two-tailed (i.e., cluster- $p$ for each tail $<0.003$ ). For each participant, the mean parameter estimates of significant clusters were extracted to visualize statistically significant results.

\section{Exploratory integrated analysis}

All baseline correlates and early changes associated with MADRS improvement were inputted in a multiple linear regression model to determine the independent associations of each of these findings with MADRS improvement. Site, sex, age, and baseline MADRS severity were inputted as covariates, and only main effects were investigated.

\section{RESULTS}

Demographic and clinical results

Age, sex, and handedness did not differ among the MDD and HC groups, nor did these demographic variables differ between recruitment sites (Table 1; Supplementary Tables 1 and 2). The mean percent improvement in the MDD group on the MADRS from baseline to week 8 was $49.5 \pm 30.1 \%$ (Fig. 1a). Mean MADRS scores in the $\mathrm{HC}$ group did not change significantly from baseline to week $8(t(42)=-0.75, p=0.46)$. Demographic and clinical results separated by categorical treatment status (response defined as $\geq 50 \%$ MADRS improvement at week 8 ) are summarized in Supplementary Results.

We performed a partial correlation, controlling for age, sex, and recruitment site, to determine whether baseline scores or week 2 percent improvement on the MADRS predicted week 8 improvement. Baseline MADRS score did not significantly correlate with

\section{A. MADRS}

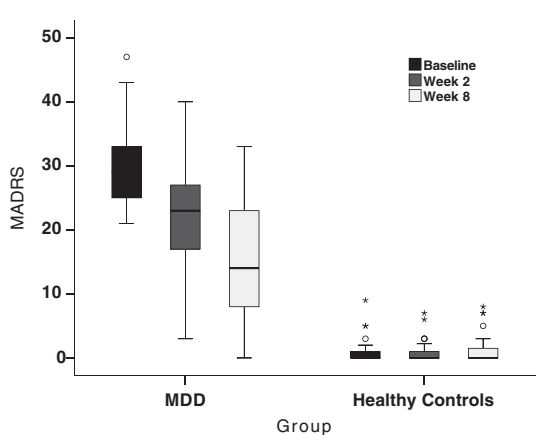

B. DARS

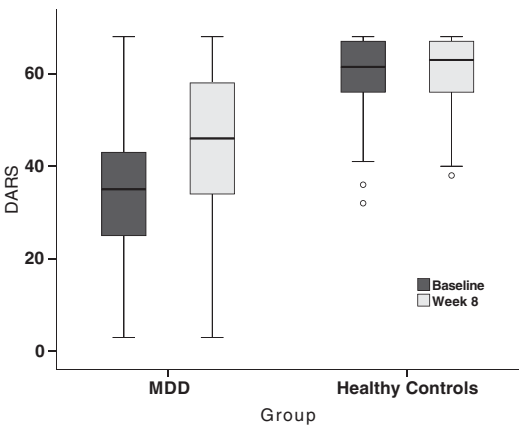

C. SHAPS

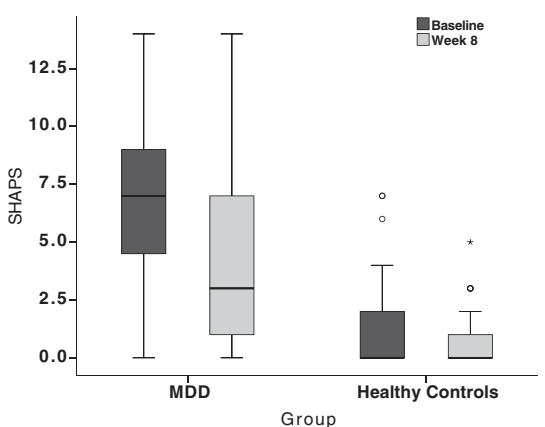

Fig. 1 Box plots representing depression and anhedonia severity for MDD and HC groups. a MADRS improvement at baseline, week 2 and week 8. b DARS improvement at baseline and week 8. c SHAPS improvement at baseline and week 8 . The hinges of the box plots represent the first quartile to the third quartile; whiskers represent the $95 \%$ confidence interval, with the exception of outliers (scores that fall outside of the $95 \%$ confidence interval; circles) and extreme outliers (scores that are three times the height of box; asterisks). 
antidepressant improvement at 8 weeks $(r=-0.065 \pm 0.11$, FDRcorrected- $p=0.56, \mathrm{df}=81,95 \% \mathrm{Cl}:-0.28,0.16)$. There was a strong correlation, however, between the percent change from baseline to week 2 and the percent change at 8 weeks, such that early improvement in MADRS at week 2 correlated with greater MADRS improvement at week $8(r=0.46 \pm 0.09$, FDR-corrected $p=0.002, \mathrm{df}=82,95 \% \mathrm{Cl}: 0.27,0.61)$.

After controlling for age, sex, and recruitment site, only baseline DARS scores significantly correlated with change in MADRS score (Fig. 1b, c), such that less severe baseline DARS scores significantly correlated with greater MADRS percent improvement at week 8 $(r=0.27 \pm 0.10$, FDR-corrected $p=0.04, \mathrm{df}=81,95 \% \mathrm{Cl}: 0.07$, 0.47). Baseline SHAPS, however, failed to reach significance prior to correcting for multiple comparisons $(r=-0.14 \pm 0.10$, uncorrected $p=0.053, \mathrm{df}=81,95 \% \mathrm{Cl}:-0.32,0.05)$.

MID task behavioral results

There were no significant differences in baseline RT between the MDD and HC groups (Supplementary materials). After controlling for age, sex and recruitment site and for multiple comparisons, faster RT at baseline was associated with greater week 8 antidepressant improvement for non-incentivized miss trials $(r=-0.27 \pm 0.11 \mathrm{SE}[95 \% \mathrm{Cl}:-0.47,-0.05]$, FDR-corrected $p=0.04$ ) but no other trial conditions (Incentivized Hits $r=$ $-0.24 \pm 0.12$ SE [95\% Cl: $-0.45,-0.002]$, FDR-corrected- $p=0.06$; Incentivized Misses $r=-0.21 \pm 0.12 \mathrm{SE}$ [95\% Cl: $-0.43,0.03$, FDR-corrected $p=0.07$; Non-Incentivized Hits $r=-0.22 \pm 0.12$ SE [95\% Cl: $-0.44,-0.02]$, FDR-corrected $p=0.07)$.

Table 2. Significant clusters during the MID task that correlated with MADRS improvement. Early changes (baseline to week 2) in ventral striatum functional connectivity during reward anticipation that correlated with later (week 8) MADRS improvement.

Region $\quad$ \# voxels cluster-p $z$-max $\frac{\mathrm{MNI}}{\frac{x \quad y \quad z}{}}$

Ventral striatum gPPI correlated to MADRS improvement: incentivized $>$ non-incentivized cues

\begin{tabular}{lllllll} 
Bilateral rostral ACC (BA32) & 286 & 0.0007 & 4.33 & -10 & 52 & 6 \\
\hline
\end{tabular}

$A C C$ anterior cingulate cortex, BA Brodmann area, gPPI generalized psychophysiological interaction, $M N I$ Montreal Neurological Institute.
Baseline and week $2 \mathrm{fMRI}$ results

The baseline anticipatory reward contrast revealed a robust network of higher and lower activation to the incentivized $>$ nonincentivized cue and rewarded $>$ neutral feedback contrasts in both MDD and HC participants (Supplementary Materials). There were no significant pre-treatment differences with respect to BOLD activity or VS-FC during reward anticipation or reward consumption between MDD and $\mathrm{HC}$ groups. The anticipatory and consummatory reward contrasts at week 2 revealed a similarly robust network of higher and lower activation to incentivized cues in both MDD and HC participants (Supplementary Materials). Similar to baseline, there were no significant differences with respect to BOLD activity or VSFC during reward anticipation or consumption between MDD and $\mathrm{HC}$ groups at week 2. There was no significant mean change in BOLD response and VS-FC during the anticipatory or consummatory reward phases of the MID task between baseline and week 2 .

fMRI correlation with MADRS improvement

BOLD response and VS-FC during the anticipatory and consummatory reward phases of the MID task at baseline did not significantly correlate with change in MADRS at 8 weeks. Among MDD participants who completed both baseline and week 2 neuroimaging $(n=81)$, only a change in frontostriatal connectivity during reward anticipation between baseline and week 2 significantly correlated with percent MADRS improvement at week 8 (Table 2 Fig. 2). Early increases in VS-FC to the bilateral rostral anterior ACC were positively correlated with baseline to week 8 MADRS improvement $(r=0.39 \pm 0.09$ SE (95\% Cl: $0.20,0.55), p=$ 0.0003 ). This finding was not significantly correlated with baseline to week 2 changes in framewise displacement $(p>0.09)$.

Post-Hoc fMRI correlation with DARS improvement Interestingly, baseline to week 2 increases in frontostriatal connectivity did not significantly correlate with baseline to week 8 DARS change $(\rho=0.13, p=0.28, \mathrm{df}=72,95 \% \mathrm{Cl}:-0.06,0.33)$ but did with baseline to week 8 SHAPS $(\rho=-0.23, p=0.045$, $\mathrm{df}=$ $72,95 \% \mathrm{Cl}:-0.42,-0.04)$. SHAPS and DARS change were highly correlated $(\rho=-0.40, p=0.0004, \mathrm{df}=73,95 \% \mathrm{Cl}:-0.60,-0.16)$, with the trend such that baseline to week 8 improvements in anhedonia were correlated with early increases in frontostriatal connectivity during reward anticipation.

Exploratory integrated analysis

A multiple linear regression model was used to determine the independent contributions of baseline and early change predictors

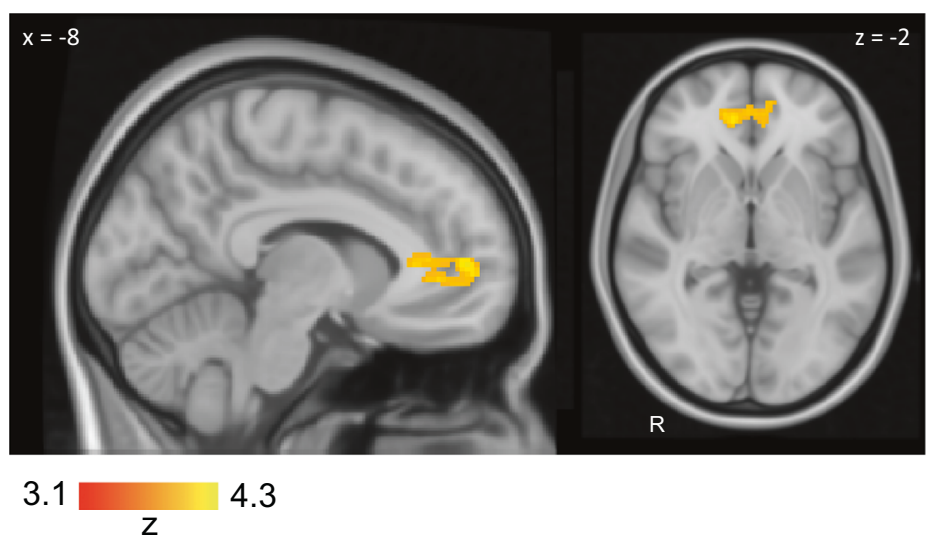

B.

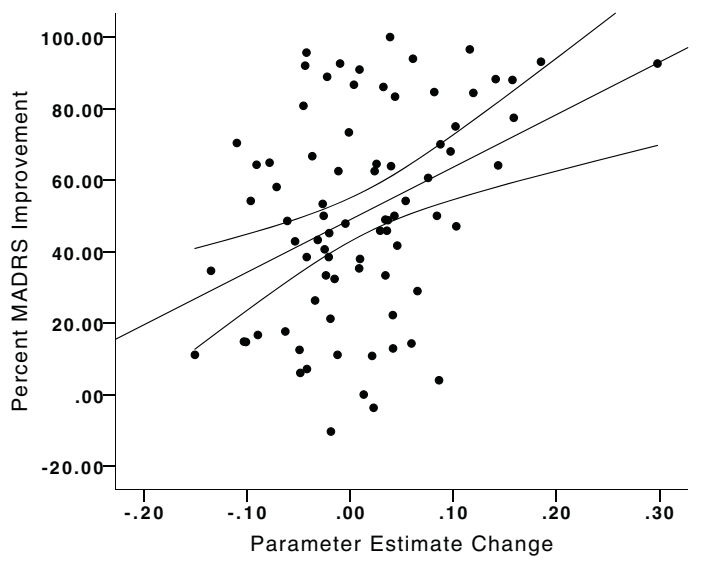

Fig. 2 Early (baseline to week 2) Increases in VS-rostral ACC Connectivity Correlates with Week 8 Escitalopram Improvement. a Increased functional connectivity in the MDD group $(n=82)$ during reward anticipation from the bilateral ventral striatum to the rostral ACC (orange-yellow) significantly correlates with MADRS improvement. b Correlation of MADRS improvement with the change in VS functional connectivity during reward anticipation from baseline to week 2 . Percent MADRS improvement was calculated such that a value of $100 \%$ represented a full improvement at week 8 (i.e., week 8 MADRS $=0$ ). 
of week 8 MADRS improvement. Main effects for baseline DARS score, RT during incentivized misses, early (baseline to week 2) MADRS improvement and parameter estimates representing early change in frontostriatal connectivity during reward anticipation were including in this model (full model $R^{2}=0.44$ ). In this model, early MADRS improvement (estimate $=0.38,95 \% \mathrm{Cl}: 0.11,0.17, t=$ $3.64, p=0.0005)$, early frontostriatal connectivity change during reward anticipation (estimate $=0.44,95 \% \mathrm{Cl}$ : 0.23, 0.64, $t=4.30, p=$ 0.0001 ), and age (estimate $=0.26,95 \% \mathrm{Cl}: 0.05,0.46, t=2.50, p=$ 0.02 ) were significantly associated with week 8 MADRS improvement. Baseline DARS (estimate $=0.06,95 \% \mathrm{Cl}:-0.15,0.27, t=0.56$, $p=0.58$ ) and baseline RT (estimate $=-0.06,95 \% \mathrm{Cl}:-0.26,0.15$, $t=-0.55, p=0.59$ ) were not significant in this model. Variance inflation factor ranged between 1.04 and 1.17, indicating low collinearity between variables.

\section{DISCUSSION}

This study aimed to examine the relations between reward processing and response to antidepressant treatment using clinical, behavioral, and functional neuroimaging measures. In 87 individuals treated with escitalopram, clinical correlates of reward processing were assessed at baseline and a MID task during functional neuroimaging was completed at baseline and after 2 weeks of treatment. The principal finding is that lower baseline anhedonia severity, as assessed by the DARS, and faster baseline RT during one MID task condition correlated with MADRS improvement after 8 weeks of escitalopram. Furthermore, early increases in the functional connectivity between the VS and rACC during the reward anticipation phase of the MID task were associated with escitalopram improvement at 8 weeks. Contrary to our other hypotheses, neither baseline BOLD activity, VS-FC during reward anticipation nor during reward consumption correlated with week 8 MADRS improvement. To our knowledge, the present study is the first to report clinical, behavioral, and neural dimensions of reward processing that correlate with escitalopram response.

The results of the present study suggest that the presence of severe pre-treatment anhedonia may indicate the earlier use of adjunctive pharmacotherapies or alternative interventions. Consistent with these findings, others have observed that high baseline anhedonia and poor reward learning were associated with poor symptomatic improvements following acute $[1,3,16,37]$ and longterm monotherapies [2]. Notably, many $[2,3,16]$ of these previous studies involving a clinical measure of anhedonia or reward processing rely on a post-hoc composite 'anhedonia score', based on an individual item from a depression severity measure such as the Beck Depression Inventory [38] or Hamilton Rating Scale for Depression [39]. In sum, the findings of the present study support the use of measures that specifically assess reward processing across dimensions (clinically, behaviorally, and based on neuroimaging tasks) in the search for predictors of response to pharmacotherapies.

Of note, the baseline scores on the DARS significantly correlated with escitalopram response, while there was a similar trend on the SHAPS. Conversely, SHAPS improvement correlated with frontostriatal change associated with escitalopram response. A validation study of the DARS found a strong correlation between DARS and SHAPS, and between the DARS and depression severity in MDD [28]. Unlike the SHAPS, DARS also includes questions related to interest, effort and motivation across four sensory domains (hobbies/activities, food/drink, social, and sensory). Furthermore, the DARS is both qualitative and quantitative: for example, participants first identify at least two of their own favorite activities or sensations and rate their immediate desire for or ability to enjoy those specific experiences. The inclusion of these personalized items assessing motivation and effort, in addition to consummatory pleasure, in the DARS may represent a more sensitive baseline measure to evaluate anhedonia as a marker of escitalopram response. However, SHAPS improvements correlated more strongly with frontostriatal increases during reward anticipation. Future work is needed to disentangle the longitudinal and independent relationships of the SHAPS and DARS with antidepressant response, particularly with respect to underlying changes in neuronal function.

Unlike previous studies $[16,17,19]$, we were unable to detect a significant correlation between baseline fMRI activity during the MID task and treatment response. In contrast, stronger baseline frontostriatal function during a reward task has been observed and related to psychotherapy response in three previous reports. In the first report, anticipation during the MID task was associated with favorable response to psychotherapy [17]; in the second, higher baseline striatal and decreased medial prefrontal activity during both reward anticipation and the receipt of rewards was associated with clinical improvement with psychotherapy [19]; and in the third, sustained baseline ACC hyperactivity during reward receipt in the MID task was noted in those who responded to psychotherapy [16]. It is possible that baseline differences in reward processing during the MID task specifically predict responsivity to psychotherapy, but not to pharmacological interventions.

Behaviorally, there was a general trend toward faster baseline RT during the MID task correlating with antidepressant improvements. Previous studies also provide evidence that faster RT during reward processing is associated with favorable psychotherapy outcomes [16]. Faster RT may indicate more motivated responding during the task [11]. Furthermore, in a large, multi-site study assessing biological subtypes of MDD reported that high anhedonia and psychomotor retardation together were associated with a distinct pattern of abnormal resting-state functional connectivity [15]. Interestingly, this association between anhedonia and psychomotor retardation identified in this neurobiological dimension provides support for our findings that both selfreported anhedonia and RTs are independently associated with escitalopram improvement.

We also observed that both early improvements in depression severity and increased frontostriatal connectivity after 2 weeks correlated with escitalopram improvement at 8 weeks; both of these findings survived in a multiple linear regression predicting escitalopram improvement at 8 weeks. This finding aligns with previously published work by our group reporting early improvements in depression severity and reward processing and early increases in oscillatory power as predictors of escitalopram response $[23,25,40]$, and the notion that neurobiological measures of anhedonia and reward processing are mediated by serotonin function [14]. Furthermore, in two meta-analyses, early clinical improvement was associated with antidepressant response $[41,42]$. Similarly, in independent studies, early neurobiological increases in rACC function within the first 2 weeks of treatment correlated with response to non-invasive brain stimulation [43], as well as response to an antidepressant in participants diagnosed with late-life depression [44]. In a subsequent study by the same group, early increases in rACC connectivity, measured by restingstate electroencephalography, predicted treatment-nonspecific remission by week 8 [24]. To our knowledge, the current study is the first to show that early increases in rACC connectivity during reward anticipation is associated with later antidepressant response.

An integrated post-hoc analysis revealed that early symptom reduction and frontostriatal change during reward anticipation each independently predicted later antidepressant improvement with escitalopram. A number of previous trials have reported early symptomatic improvements irrespective of treatment arm [45-48], potentially obscuring the true antidepressant or biological effects of the drug and impeding signal detection. Future work should aim to disentangle the independent contributions of true 
antidepressant and placebo responses during the early stages of treatment, and their relationships with neurobiological change.

We did not observe significant baseline or week 2 group differences in task-evoked BOLD activity, even though the mean activations of both the $\mathrm{HC}$ and MDD groups during reward anticipation correspond with previous publications of the MID task $[49,50]$. However, the fact that these two groups did not significantly differ is in contrast to some [11] but not all $[16,30]$ prior reports. Two possibilities could explain these differences. First, the MID task design of the current study did not include losses or changes in the magnitude of monetary gains, which have been previously shown to dissociate HC from MDD participants [30, 51]. Second, a previous study showed that genetic polymorphisms related to dopaminergic function were associated with inter-individual variability during the MID task in healthy volunteers [52]. Future analyses within CAN-BIND platforms are planned to integrate task-based neuroimaging with genetic polymorphism data.

The study has several limitations. The predictors we report here are derived from a single sample and require confirmation in an independent cohort. This study did not include a placebo arm, since the primary study aim was not to establish the efficacy of escitalopram, but rather to distinguish the phenotype of responders vs. non-responders to this common treatment. While the total sample size was relatively large, the various potential clinical subgroups of MDD may not have been adequately powered to detect subtle changes in behavioral or imaging measures.

In conclusion, we found that baseline clinical and behavioral measures of reward processing, in addition to an early change in depression severity and frontostriatal connectivity during reward anticipation, were each significantly associated with antidepressant response. Importantly, these differences remained significant even after co-varying for recruitment site, age, sex, and baseline severity. In an integrated post-hoc analysis, early symptom and frontostriatal change during reward anticipation each independently predicted later escitalopram improvement. These results are an important step toward characterizing the role of anhedonia and its biological underpinnings in MDD, including understanding whether anhedonia has a role in predicting response to treatment.

\section{FUNDING AND DISCLOSURE}

This research was conducted as part of the CAN-BIND, an Integrated Discovery Program supported by the Ontario Brain Institute, which is an independent non-profit corporation, funded partially by the Ontario government. The opinions, results, and conclusions are those of the authors and no endorsement by the Ontario Brain Institute is intended or should be inferred. Additional funding was provided by the Canadian Institutes of Health Research (CIHR), the National Science and Engineering Council of Canada (NSERC), Lundbeck, Bristol-Myers Squibb, Pfizer, and Servier. Funding and/or in-kind support was also provided by the investigators' universities and academic institutions. All study medications were independently purchased at wholesale market values. KD reports grants from CIHR - Banting \& Vanier Fellowships, outside the submitted work. SJR reports grants from CIHR during the conduct of the study; grants from Brain and Behavior Foundation, personal fees from Pfizer Canada, personal fees from Janssen, personal fees from Quintiles, grants from St. Michael's Hospital Foundation, outside the submitted work. SHK reports grants from Lundbeck, Bristol-Myers Squibb, Pfizer, Servier, Canadian Institutes for Health Research (CIHR), during the conduct of the study; and Abbott; personal fees from Alkermes, grants from Allergan, grants and personal fees from Janssen, grants and personal fees from Lundbeck, personal fees from Lundbeck Institute, grants and personal fees from Otsuka, personal fees from Pfizer, personal fees from Servier, outside the submitted work. SH has nothing to disclose. SCS reports other from ADM
Diagnostics, LLC, Chicago, outside the submitted work. JKH, MZ, SRA, ADD have nothing to disclose. FM reports grants from Mitacs, outside the submitted work. LS reports grants from Ontario Mental Health Foundation, outside the submitted work. AKC has nothing to disclose. RWL reports grants from Lundbeck, grants from Pfizer, grants from Canadian Institutes for Health Research (CIHR), during the conduct of the study; personal fees from Akili, personal fees from Allergan, personal fees from Asia-Pacific Economic Cooperation, personal fees from AstraZeneca, personal fees from BC Leading Edge Foundation, personal fees from Canadian Network for Mood and Anxiety Treatments, personal fees from Canadian Psychiatric Association, personal fees from CME Institute, personal fees from Hansoh, personal fees from Healthy Minds Canada, personal fees from Lundbeck Institute, personal fees from Janssen, personal fees from Medscape, personal fees from Mind.Me, grants from MITACS, personal fees from Movember Foundation, personal fees from Otsuka, personal fees from St. Jude Medical, grants from University Health Network Foundation, grants from VGH-UBCH Foundation, outside the submitted work. RM reports grants from $\mathrm{ClHR}$, grants from Janssen, grants from Lundbeck, during the conduct of the study; personal fees from Allergan, personal fees from Janssen, personal fees from Kye Pharmaceuticals, personal fees from Lundbeck, personal fees from Otsuka, grants and personal fees from Pfizer, personal fees from Sunovion, grants from Lallemand, grants from Nubiyota, grants from Ontario Mental Health Foundation, outside the submitted work. SR and JAF have nothing to disclose. BNF reports grants from CIHR, during the conduct of the study; grants from Pfizer, outside the submitted work. SVP reports grants from CIHR, during the conduct of the study; personal fees from Takeda, personal fees from Bristik Myers Squibb, personal fees from Lundbeck, personal fees from Assurex, other from Mensante, outside the submitted work. CNS and RU have nothing to disclose. GT reports personal fees from Pfizer Canada, personal fees from Bristol-Meyers Squibb, from Janssen Canada, outside the submitted work. GMM reports personal fees and other from Lundbeck, other from Lilly, personal fees and other from Pfizer, personal fees and other from Janssen, personal fees from AstraZeneca, personal fees from Bristol-Myers Squibb, grants from ClHR, personal fees from Canadian Network for Mood and Anxiety Treatments, personal fees from Eli Lilly, grants from Ontario Mental Health Foundation, personal fees from Otsuka, personal fees from Paladin, personal fees from Sunovion, grants from University Health Network Foundation, outside the submitted work. JD reports grants from CIHR, during the conduct of the study; grants from National Institutes of Health, grants from Brain Canada, grants from Klarman Family Foundation, grants from Edgestone Foundation, grants from Toronto General and Western Hospital Foundation, personal fees from Lundbeck, personal fees from ANT Neuro, non-financial support from MagVenture, other from BrainCheck, other from Restorative Brain Clinics, other from TMS Neuro Solutions, outside the submitted work.

\section{ACKNOWLEDGEMENTS}

We are indebted to all participants and clinicians for the time and effort they dedicated to this research. We thank Dr Diego Pizzagalli (Harvard Medical School, Belmont, MA, USA) for consultation and assistance with study design. We also thank Keith Ho (University Health Network, Toronto, Ontario, Canada) and Zahra Moazamigoudarzi (St. Michael's Hospital, Toronto, Ontario, Canada) for their assistance in preparation of data for this manuscript. The authors wish to thank all members of the CAN-BIND Investigator team for their contribution to this work. Members of the CAN-BIND Investigator Team are listed here: www.canbind.ca/our-team/.

\section{AUTHOR CONTRIBUTIONS}

KD: Data curation, formal analysis, writing (original draft), writing (review, editing, and approval); SJR: formal analysis, writing (original draft), writing (review, editing \& approval); SHK: Study concept, data collection, formal analysis, writing (review, 
editing, and approval); SH: Data collection, data curation, writing (review, editing \& approval); SCS: Data curation, writing (review, editing \& approval); JKH: Data collection, data curation, writing (review, editing, and approval); MZ: Data collection, data curation, writing (review, editing, and approval); SRA: Data curation, writing (review, editing, and approval); ADD: Data collection, data curation, writing (review, editing, and approval); FM: Formal analysis, writing (review, editing, and approval); LS: Formal analysis, writing (review, editing, and approval); AKC: Data collection, data curation, writing (review, editing, and approval); RWL: Study concept, data collection, writing (review, editing \& approval); RM: Study concept, data collection, writing (review, editing \& approval); SR: Study concept, data collection, data curation, writing (review, editing, and approval); JAF: Data collection, data curation, writing (review, editing, and approval); BNF: Study concept, data collection, data curation, writing (review, editing, and approval); SVP: Study concept, writing (review, editing, and approval); CNS: Data collection, data curation, writing (review, editing, and approval); RU: Writing (review, editing, and approval); GT: Writing (review, editing and approval); GMM: Study concept, data collection, data curation, writing (review, editing, and approval); JD: Data collection, data curation, formal analysis, writing (review, editing, and approval)

\section{ADDITIONAL INFORMATION}

Supplementary Information accompanies this paper at (https://doi.org/10.1038/ s41386-020-0688-x).

Publisher's note Springer Nature remains neutral with regard to jurisdictional claims in published maps and institutional affiliations.

\section{REFERENCES}

1. Vrieze E, Pizzagalli DA, Demyttenaere K, Hompes $T$, Sienaert $P$, de Boer $P$, et al. Reduced reward learning predicts outcome in major depressive disorder. Biol Psychiatry. 2013;73:639-45.

2. Spijker J, Bijl RV, de Graaf R, Nolen WA. Determinants of poor 1-year outcome of DSM-III-R major depression in the general population: results of the Netherlands Mental Health Survey and Incidence Study (NEMESIS). Acta Psychiatr Scand. 2001;103:122-30.

3. Downar J, Geraci J, Salomons TV, Dunlop K, Wheeler S, McAndrews MP, et al. Anhedonia and reward-circuit connectivity distinguish nonresponders from responders to dorsomedial prefrontal repetitive transcranial magnetic stimulation in major depression. Biol Psychiatry. 2014;76:176-85.

4. Warden D, Rush AJ, Trivedi MH, Fava M, Wisniewski SR. The STAR*D Project results: a comprehensive review of findings. Curr Psychiatry Rep. 2007;9: 449-59.

5. Rush AJ, Trivedi MH, Wisniewski SR, Nierenberg AA, Stewart JW, Warden D, et al. Acute and longer-term outcomes in depressed outpatients requiring one or several treatment steps: a STAR*D report. Am J Psychiatry. 2006;163:1905-17.

6. Hägele C, Schlagenhauf F, Rapp M, Sterzer P, Beck A, Bermpohl F, et al. Dimensional psychiatry: reward dysfunction and depressive mood across psychiatric disorders. Psychopharmacology. 2015;232:331-41.

7. Dubol M, Trichard C, Leroy C, Sandu A-L, Rahim M, Granger B, et al. Dopamine transporter and reward anticipation in a dimensional perspective: a multimodal brain imaging study. Neuropsychopharmacology. 2018;43:820-7.

8. Stoy $M$, Schlagenhauf $F$, Sterzer $P$, Bermpohl $F$, Hägele $C$, Suchotzki $K$, et al. Hyporeactivity of ventral striatum towards incentive stimuli in unmedicated depressed patients normalizes after treatment with escitalopram. J Psychopharmacol. 2012;26:677-88.

9. Dichter GS, Kozink RV, McClernon FJ, Smoski MJ. Remitted major depression is characterized by reward network hyperactivation during reward anticipation and hypoactivation during reward outcomes. J Affect Disord. 2012;136:1126-34.

10. Zhang B, Lin P, Shi H, Öngür D, Auerbach RP, Wang X, et al. Mapping anhedoniaspecific dysfunction in a transdiagnostic approach: an ALE meta-analysis. Brain Imaging Behav. 2016;10:920-39.

11. Pizzagalli DA, Holmes AJ, Dillon DG, Goetz EL, Birk JL, Bogdan R, et al. Reduced caudate and nucleus accumbens response to rewards in unmedicated individuals with major depressive disorder. Am J Psychiatry. 2009;166:702-10.

12. Admon R, Nickerson LD, Dillon DG, Holmes AJ, Bogdan R, Kumar P, et al. Dissociable cortico-striatal connectivity abnormalities in major depression in response to monetary gains and penalties. Psychol Med. 2015;45:121-31.

13. Wacker J, Dillon DG, Pizzagalli DA. The role of the nucleus accumbens and rostral anterior cingulate cortex in anhedonia: integration of resting EEG, FMRI, and volumetric techniques. Neuroimage. 2009;46:327-37.

14. Prakash N, Stark CJ, Keisler MN, Luo L, Der-Avakian A, Dulcis D. Serotonergic plasticity in the dorsal raphe nucleus characterizes susceptibility and resilience to anhedonia. J Neurosci. 2020;40:569-84.
15. Drysdale AT, Grosenick L, Downar J, Dunlop K, Mansouri F, Meng Y, et al. Restingstate connectivity biomarkers define neurophysiological subtypes of depression. Nat Med. 2017;23:28-38.

16. Carl H, Walsh E, Eisenlohr-Moul T, Minkel J, Crowther A, Moore T, et al. Sustained anterior cingulate cortex activation during reward processing predicts response to psychotherapy in major depressive disorder. J Affect Disord. 2016;203:204-12.

17. Walsh E, Carl H, Eisenlohr-Moul T, Minkel J, Crowther A, Moore T, et al. Attenuation of frontostriatal connectivity during reward processing predicts response to psychotherapy in major depressive disorder. Neuropsychopharmacology. 2017;42:831-43.

18. Kujawa A, Burkhouse KL, Karich SR, Fitzgerald KD, Monk CS, Phan KL. Reduced reward responsiveness predicts change in depressive symptoms in anxious children and adolescents following treatment. J Child Adolesc Psychopharmacol. 2019;29:378-85.

19. Forbes EE, Olino TM, Ryan ND, Birmaher B, Axelson D, Moyles DL, et al. Rewardrelated brain function as a predictor of treatment response in adolescents with major depressive disorder. Cogn Affect Behav Neurosci. 2010;10:107-18.

20. Admon R, Kaiser RH, Dillon DG, Beltzer M, Goer F, Olson DP, et al. Dopaminergic enhancement of striatal response to reward in major depression. Am J Psychiatry. 2017;174:378-86.

21. Cheng $Y, X u$ J, Arnone $D, N i e ~ B, Y u ~ H$, Jiang $H$, et al. Resting-state brain alteration after a single dose of SSRI administration predicts 8-week remission of patients with major depressive disorder. Psychol Med. 2017;47:438-50.

22. Burkhouse $\mathrm{KL}$, Gorka $\mathrm{SM}$, Klumpp $\mathrm{H}$, Kennedy $\mathrm{AE}$, Karich $\mathrm{S}$, Francis J, et al. Neural responsiveness to reward as an index of depressive symptom change following cognitive-behavioral therapy and SSRI treatment. J Clin Psychiatry. 2018;79:17m11836.

23. Allen TA, Lam RW, Milev R, Rizvi SJ, Frey BN, MacQueen GM, et al. Early change in reward and punishment sensitivity as a predictor of response to antidepressant treatment for major depressive disorder: a CAN-BIND-1 report. Psychol Med. 2019;49:1629-38.

24. Whitton AE, Webb CA, Dillon DG, Kayser J, Rutherford A, Goer F, et al. Pretreatment rostral anterior cingulate cortex connectivity with salience network predicts depression recovery: findings from the EMBARC randomized clinical trial. Biol Psychiatry 2019;85:872-80.

25. Kennedy SH, Lam RW, Rotzinger S, Milev RV, Blier P, Downar J, et al. Symptomatic and functional outcomes and early prediction of response to escitalopram monotherapy and sequential adjunctive aripiprazole therapy in patients with major depressive disorder: a CAN-BIND-1 report. J Clin Psychiatry. 2019;80:18m12202.

26. Montgomery SA, Asberg M. A new depression scale designed to be sensitive to change. Br J Psychiatry. 1979;134:382-9.

27. Snaith RP, Hamilton M, Morley S, Humayan A, Hargreaves D, Trigwell P. A scale for the assessment of hedonic tone the Snaith-Hamilton Pleasure Scale. Br J Psychiatry. 1995;167:99-103.

28. Rizvi SJ, Quilty LC, Sproule BA, Cyriac A, Michael Bagby R, Kennedy SH. Development and validation of the Dimensional Anhedonia Rating Scale (DARS) in a community sample and individuals with major depression. Psychiatry Res. 2015;229:109-19.

29. Alders GL, Davis AD, MacQueen G, Strother SC, Hassel S, Zamyadi M, et al. Reduced accuracy accompanied by reduced neural activity during the performance of an emotional conflict task by unmedicated patients with major depression: A CAN-BIND fMRI study. J Affect Disord. 2019;257:765-73.

30. Knutson B, Bhanji JP, Cooney RE, Atlas LY, Gotlib IH. Neural responses to monetary incentives in major depression. Biol Psychiatry. 2008;63:686-92.

31. Schiller CE, Minkel J, Smoski MJ, Dichter GS. Remitted major depression is characterized by reduced prefrontal cortex reactivity to reward loss. J Affect Disord. 2013;151:756-62.

32. Smoski MJ, Rittenberg A, Dichter GS. Major depressive disorder is characterized by greater reward network activation to monetary than pleasant image rewards. Psychiatry Res. 2011;194:263-70.

33. MacQueen GM, Hassel S, Arnott SR, Jean A, Bowie CR, Bray SL, et al. The Canadian Biomarker Integration Network in Depression (CAN-BIND): magnetic resonance imaging protocols. J Psychiatry Neurosci. 2019;44:223-36.

34. Jenkinson M, Beckmann CF, Behrens TEJ, Woolrich MW, Smith SM. FSL. Neuroimage 2012;62:782-90.

35. Costumero V, Barrós-Loscertales A, Bustamante JC, Ventura-Campos N, Fuentes P, Ávila C. Reward sensitivity modulates connectivity among reward brain areas during processing of anticipatory reward cues. Eur J Neurosci. 2013;38:2399-407.

36. Desikan RS, Ségonne F, Fischl B, Quinn BT, Dickerson BC, Blacker D, et al. An automated labeling system for subdividing the human cerebral cortex on MRI scans into gyral based regions of interest. Neuroimage. 2006;31:968-80.

37. Uher R, Perlis RH, Henigsberg N, Zobel A, Rietschel M, Mors O, et al. Depression symptom dimensions as predictors of antidepressant treatment outcome: replicable evidence for interest-activity symptoms. Psychol Med. 2012;42:967-80.

38. Beck AT, Steer RA, Ball R, Ranieri W. Comparison of Beck Depression Inventories -IA and -II in psychiatric outpatients. J Pers Assess. 1996;67:588-97. 
39. Hamilton MC. A rating scale for depression. J Neurol Neurosurg Psychiatry. 1960; 23:56-62.

40. Baskaran A, Farzan F, Milev R, Brenner CA, Alturi S, Pat McAndrews M, et al. The comparative effectiveness of electroencephalographic indices in predicting response to escitalopram therapy in depression: a pilot study. J Affect Disord. 2018;227:542-9.

41. Wagner S, Engel A, Engelmann J, Herzog D, Dreimüller N, Müller MB, et al. Early improvement as a resilience signal predicting later remission to antidepressant treatment in patients with Major Depressive Disorder: Systematic review and meta-analysis. J Psychiatr Res. 2017;94:96-106.

42. Olgiati P, Serretti A, Souery D, Dold M, Kasper S, Montgomery S, et al. Early improvement and response to antidepressant medications in adults with major depressive disorder. Meta-analysis and study of a sample with treatmentresistant depression. J Affect Disord. 2018;227:777-86.

43. Hunter AM, Nghiem TX, Cook IA, Krantz DE, Minzenberg MJ, Leuchter AF. Change in quantitative EEG theta cordance as a potential predictor of repetitive transcranial magnetic stimulation clinical outcome in major depressive disorder. Clin EEG Neurosci. 2018;49:306-15.

44. Karim HT, Wang M, Andreescu C, Tudorascu D, Butters MA, Karp JF, et al. Acute trajectories of neural activation predict remission to pharmacotherapy in late-life depression. Neurolmage Clin. 2018;19:831-9.

45. Targum SD. Early symptomatic improvement affects treatment outcome in a study of major depressive disorder. J Psychiatr Res. 2017;95:276-81.
46. Khan A, Cohen S, Dager S, Avery DH, Dunner DL. Onset of response in relation to outcome in depressed outpatients with placebo and imipramine. J Affect Disord. 1989;17:33-8.

47. Quitkin FM, Rabkin JG, Ross D, Stewart JW. Identification of true drug response to antidepressants. Use of pattern analysis. Arch Gen Psychiatry. 1984;41: 782-6.

48. Kemp DE, Ganocy SJ, Brecher M, Carlson BX, Edwards S, Eudicone JM, et al. Clinical value of early partial symptomatic improvement in the prediction of response and remission during short-term treatment trials in 3369 subjects with bipolar I or II depression. J Affect Disord. 2011;130:171-9.

49. Knutson B, Westdorp A, Kaiser E, Hommer D. FMRI visualization of brain activity during a monetary incentive delay task. Neuroimage. 2000;12:20-7.

50. Wilson RP, Colizzi M, Bossong MG, Allen P, Kempton M, MTAC, et al. The neural substrate of reward anticipation in health: a meta-analysis of $f M R I$ findings in the monetary incentive delay task. Neuropsychol Rev. 2018;28: 496-506.

51. Sankar A, Yttredahl AA, Fourcade EW, Mickey BJ, Love TM, Langenecker SA, et al. Dissociable neural responses to monetary and social gain and loss in women with major depressive disorder. Front Behav Neurosci. 2019;13:149.

52. Dillon DG, Bogdan R, Fagerness J, Holmes AJ, Perlis RH, Pizzagalli DA Variation in TREK1 gene linked to depression-resistant phenotype is associated with potentiated neural responses to rewards in humans. Hum Brain Mapp. 2010;31:210-21. 